\title{
021
}

\section{MICOPLASMI: \\ RISULTATI PRELIMINARI \\ DI ISOLAMENTI NELLA POPOLAZIONE GIOVANILE}

\author{
Audisio G.; 'lanotti L.; Orso Giacone G.; 'Vola M. \\ Laboratorio Analisi Collegno A.S.L. 5 (TO) \\ 'Consultori A.S.L. 5
}

Il laboratorio analisi cliniche di Collegno collabora con il laboratorio dell'Ospedale Sant'Anna di Torino ad uno studio finanziato dalla Regione Piemonte per lo studio della prevalenza da Chlamydia trachomatis., mediante autoprelievo, nella fascia di popolazione, 18-24 anni, identificata come maggiormente a rischio MST. Nel protocollo del laboratorio di Collegno le pazienti prima dell'esame batteriologico cervico-vaginale standardizzato per MST sono invitate ad effettuare due tamponcini di autoprelievo per permettere la ricerca della clamidia con le metodiche di biologia molecolare in uso nei due laboratori. Con l'occasione si è voluto porre a confronto l'isolamento di altri patogeni MST quali i micoplasmi. Il campione cervicale è stato seminato direttamente al momento del prelievo grazie alla praticità della nuova metodica MYCOFAST EvolutioN 3 commercializzata dalla DID. Il Kit permette la ricerca, la conta e l'identificazione di U.urealyticum e M.hominis. con la possibilità di effettuare test di sensibilità nei confronti di sette antibiotici. Le pazienti inviate dai consultori nel periodo settembre 2004-maggio 2005 sono state 89. M.hominis è stato isolato nel 3,37\% delle pazienti ed U.urealyticum nel $12,4 \%$ contro il 5,6\% di C. trachomatis t.. Le sensibilità rilevate: Pristinamicina $100 \%$, Ciprofloxacina 18,1\%, Azitromicina 9\%, Josamicina $100 \%$, Doxiciclina, $91 \%$, Roxitromicina $54,5 \%$, Ofloxacina $27,2 \%$.

La popolazione aveva le seguenti caratteristiche: $27 \%$ età primo rapporto inferiore ai 16 anni, $23 \%$ rapporti con partner occasionali, 33\% partner complessivi superiore a 3, 6,7\% 1 o 2 aborti, 18\% uso del condom (sempre o saltuario), $78,6 \%$ assenza di test per HIV, 7,8\% con partner sintomatico, $11,2 \%$ uso di sostanze stupefacenti. 